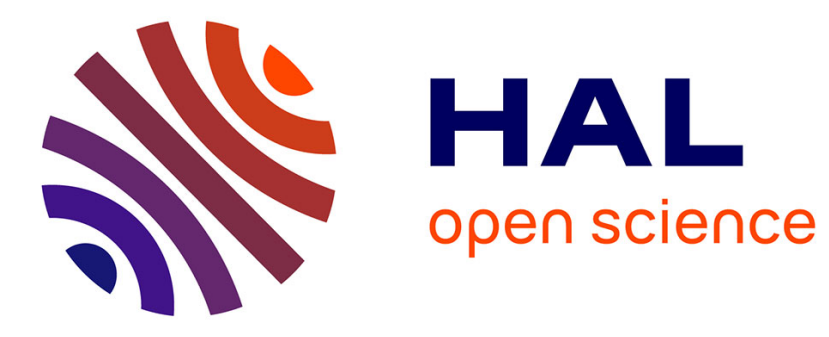

\title{
Evaluation of workers exposure to magnetic fields
}

\author{
A. Canova, F. Freschi, M. Repetto
}

\section{To cite this version:}

A. Canova, F. Freschi, M. Repetto. Evaluation of workers exposure to magnetic fields. European Physical Journal: Applied Physics, 2010, 52 (2), 10.1051/epjap/2010009466 . hal-00634373

\section{HAL Id: hal-00634373 https://hal.science/hal-00634373}

Submitted on 21 Oct 2011

HAL is a multi-disciplinary open access archive for the deposit and dissemination of scientific research documents, whether they are published or not. The documents may come from teaching and research institutions in France or abroad, or from public or private research centers.
L'archive ouverte pluridisciplinaire HAL, est destinée au dépôt et à la diffusion de documents scientifiques de niveau recherche, publiés ou non, émanant des établissements d'enseignement et de recherche français ou étrangers, des laboratoires publics ou privés. 


\title{
Evaluation of workers exposure to magnetic fields
}

\author{
Aldo Canova, Fabio Freschi, Maurizio Repetto \\ Politecnico di Torino, Dipartimento di Ingegneria Elettrica, I-10129 Torino, Italy
}

Received: date / Revised version: date

\begin{abstract}
Following ICNIRP Guidelines of 1998, European Parliament in 2004 has stated the reference levels for workers exposure to electromagnetic fields. In low frequency regime, due to the nature of the basic mechanism of short term interaction, the exposure limits are based on the values of induced eddy currents inside human body, thus electromagnetic simulation is an important tool for the assessment of electromagnetic field exposure. This paper presents a method for computing eddy currents inside human body and applies the method to the evaluation of eddy currents induced by a resistance spot welding system. A comparison between results obtained with two different models of human body and with different discretization levels is performed and results are discussed.
\end{abstract}

PACS. 87.50.C - Static and low-frequency electric and magnetic fields effects - 87.50.cm - Dosimetry/exposure assessment - 41.20.-q - Applied classical electromagnetism

\section{Introduction}

Protection of worker population against the possible effects of extremely low frequency (ELF) electromagnetic fields is a concern of European Community which has published in 2004 Directive 2004/40/EC [1] that refers to the risk to the health and safety of workers due to known short-term adverse effects in the human body caused by the circulation of induced currents and by energy absorption as well as by contact currents. The deadline for complying to the Directive was initially set at 30 April 2008 and now has been delayed of four years. Notwithstanding the enforcement of the Directive has been postponed, there is actually large interest in assessing possible actions that will have to be taken to comply with it. Actions in industrial equipments could range from the insertion of shielding systems to reduce the environmental electromagnetic field to complete redesign of apparatus.

One of the most important points stated in the Directive is the rationale of exposure definition which is defined in accordance with ICNIRP 1998 guidelines [2]. These 
Guidelines report that in the ELF frequency range, the risk to the health and safety of workers is due to known short-term adverse effects in the human body caused by the circulation of induced currents and by energy absorption as well as by contact currents. Since interest is focused on levels of induced eddy currents inside the living tissues by surrounding EMF, the main operative problem is how to assess the magnitude of the effect (eddy current) inside the body knowing the cause (the surrounding magnetic field).

As a way of tackling this issue, both ICNIRP and EU Directive define two threshold levels of physical quantities: one level related to limits on exposure to electromagnetic fields which are based directly on established health effects and biological considerations, named exposure limit value, and another based on the magnitude of directly measurable parameters, provided in terms of field strength. Compliance with these values will ensure compliance with the relevant exposure limit values. These levels are named action values in EU Directive and reference values by ICNIRP. Since both documents take into account a broad range of operating frequencies, there is not a unique threshold value but more levels which are function of frequency. As a matter of levels at the industrial frequency of $50 \mathrm{~Hz}$ the action value on magnetic flux density is set at $0.5 \mathrm{mT}[1]$.

Once the threshold levels are set, the assessment of possible problems caused by an installation requires several operative steps:
1. quantitative assessment of magnetic flux density created by devices. This can be accomplished by measurements on already existing systems or by numerical simulation for apparatuses at the design level;

2. comparison of the previously computed levels with $a c$ tion values: this action creates two possible outcomes:

(a) magnetic flux density values are lower than action values and thus no further action has to be taken;

(b) magnetic flux density values are higher than $a c$ tion values and thus compliance with exposure limit value must be assessed.

The evaluation of second point is not an easy task, involving the study of eddy currents inside a complex system like the human body. Studies at the international levels have produced technical documents that tackle this aspect which have given rise to the International Standard IEC 62226 [3]. This Standard has the aim to propose a more realistic approach to the modelling of the human exposure to low frequency electric and magnetic fields, using a set of models of growing complexity for the field emission source, or the human body or both. Crude models based on homogeneous conducting disks with electrical conductivity equal to the average value of different tissues present in the body are proposed. In case where these techniques are too simplified, Standards defines the possibility of direct computation of induced electrical quantities in the body using sophisticated computation models. Thus the use of electromagnetic analysis tools for assessing the exposure levels is possible and this fact opens an opportunity of application to this topic of numerical tools. Several dif- 
ficulties are present at this stage: modelling of external magnetic fields and of the human body are not an easy task. In the following the main simplification hypotheses that can be adopted in the computation of eddy currents are described and then the application of numerical evaluation tools to an industrial case of resistance spot welding is presented and discussed.

\section{Evaluation of induced eddy currents in}

\section{human body}

Computation of eddy currents in conducting industrial structures under magneto quasi-static hypothesis is, nowadays, a routine activity in electromagnetic analysis. Notwithstanding this consideration, the computation of induced eddy currents inside living bodies keeps some characteristic features that make the problem non standard. If assessment of dosimetric quantities is sought, the first peculiarity to be considered is tied to the large distance between magnetic field source and living being: usually the magnetic field exposure is in the region of far field and this fact makes the use of a volume meshing difficult, for instance if finite element method has to be used the discretization of air region would be needed. In this case a very large mesh must be employed and problems may arise because of a poor reconstruction of source magnetic field. In this respect, integral or hybrid techniques, which do not require the discretization of air region and correctly follow far field at infinity, seem to be preferable. At the same time, human tissues have a low value of electrical conductivity which, at low frequency, usually gives rise to a negligible reaction field. This characteristic allows to consider the eddy current problem as if it would be under impressed magnetic field distribution created by some source outside the body: electro-motive forces arise inside the body but their effects are not large enough to change the source field. In this case electro-motive forces can be computed directly from time derivative source magnetic flux.

\subsection{Scalar Potential Finite Difference}

The Scalar-Potential Finite-Differences (SPFD) method for the calculation of induced current densities inside the human body has been introduced in [4]. This approximated approach is valid when the magnetic field created by induced currents is small with respect to the one created by source currents. Under this hypothesis, the magnetic field distribution is not perturbed by eddy currents and so it can be computed independently on the conducting body. By making reference to the standard notation for discrete operators introduced in [5] and resumed in [6], the problem can be formulated in algebraic form as follows.

Solenoidality of eddy currents (since displacement currents are neglected) imposes that:

$$
\tilde{\mathbf{D}} \mathbf{i}=\mathbf{0}
$$

where $\tilde{\mathbf{D}}$ is the discrete expression of divergence operator and $\mathbf{i}$ is the vector of induced eddy currents. Electromagnetic induction law can be expressed in terms of magnetic 
vector potential:

$$
\mathbf{u}=-\mathbf{G} \varphi-\frac{\mathrm{d} \mathbf{a}}{\mathrm{d} t}
$$

where $\tilde{\mathbf{G}}$ is the discrete expression of gradient operator, $\mathbf{u}$ are induced electro-motive forces, $\varphi$ is the vector of electric scalar potential and $\mathbf{a}$ is the vector of magnetic vector potential. Electrical constitutive equation can be written as:

$$
\mathbf{i}=\mathbf{M}_{\sigma} \mathbf{u}
$$

where $\mathbf{M}_{\boldsymbol{\sigma}}$ is the discrete expression of Ohm's law. By inserting (3) in (1):

$$
\tilde{\mathbf{D}} \mathbf{M}_{\sigma} \mathbf{u}=\mathbf{0}
$$

by using (2) and duality relations $\tilde{\mathbf{D}}=-\mathbf{G}^{\mathrm{T}}[5]$ :

$$
\mathbf{G}^{\mathrm{T}} \mathbf{M}_{\boldsymbol{\sigma}} \mathbf{G} \boldsymbol{\varphi}+\mathbf{G}^{\mathrm{T}} \mathbf{M}_{\boldsymbol{\sigma}} \frac{\mathrm{d} \mathbf{a}}{\mathrm{d} t}=\mathbf{0}
$$

Finally, by exploiting the hypothesis that magnetic flux would not be modified by eddy currents, magnetic part of (4) is a known term so that previous equation can be rewritten as:

$$
\mathbf{G}^{\mathrm{T}} \mathbf{M}_{\boldsymbol{\sigma}} \mathbf{G} \boldsymbol{\varphi}=-\mathbf{G}^{\mathrm{T}} \mathbf{M}_{\boldsymbol{\sigma}} \frac{\mathrm{d} \mathbf{a}_{\mathbf{s}}}{\mathrm{d} t}
$$

where $\mathbf{a}_{\mathbf{s}}$ is the magnetic vector potential due to imposed current sources.

Under sinusoidal steady-state (with angular frequency $\omega)$, previously defined variables become phasors and (6) becomes:

$$
\mathbf{G}^{\mathrm{T}} \mathbf{M}_{\boldsymbol{\sigma}} \mathbf{G} \underline{\varphi}=-j \omega \mathbf{G}^{\mathrm{T}} \mathbf{M}_{\boldsymbol{\sigma}} \underline{\mathbf{a}}_{\mathbf{s}}
$$

\section{Modelling of human body}

The choice of a suitable human model is not a standardized task. In fact, possible 3D models may have different spatial resolutions and tissue properties are not well assessed. On the other hand, finer models introduce computational complexity due to the large amount of data to be processed, leading to slow simulation and approximate solutions. In this study two 3D models of human body are used, each with three spatial discretizations. The axisymmetrical body model represents the essential features of the body: its height, total surface area, neck dimensions, and approximate vertical profile. It is based on anthropometric measurement made on a representative sample of US army people [7]. This model is proposed IEC Standard 62226-3-1 [8] for the calculation of induced currents in presence of external electric field and it is here adopted for the similarities of SPFD formulation with that for electric field.

The second realistic model is the "Hugo" 3D anatomical data set [9]. It is a discretization of the body of a 38 year old man of about $180 \mathrm{~cm}$ height. Many resolutions are available from $8 \times 8 \times 8 \mathrm{~mm}$ down to $1 \times 1 \times 1 \mathrm{~mm}$ voxels.

Due to the fact that only industrial frequencies are considered for this study, biological tissues are characterized only by their conductivity. Displacement currents can be considered negligible in a first approximation of the problem. The axisymmetric model is considered homogeneous with uniform average conductivity of $0.2 \mathrm{~S} / \mathrm{m}$. Hugo is described by 31 different tissues, whose properties are approximated by the parametric Cole-Cole model [10] on data available in [11] 
Table 1 shows the conductivities used in this study for each tissue. The value of $\omega \epsilon$ is also reported, in order to make a direct comparison with the correspondent value of $\sigma$ and validate the hypothesis of neglecting displacement currents.

Both models have been tested with three different discretizations. The three dataset used for Hugo correspond to cubic voxels with edges of $8-6-4 \mathrm{~mm}$ respectively. The tetrahedral discretization used for the axisymmetric model are chosen in order to have the same number of cells used for Hugo. Table 2 reports these mesh data.

\section{Resistance spot welding system}

The environmental impact of a resistance spot welding system depends on the supply conditions: alternate current $(\mathrm{AC})$ and medium frequency direct current (MFDC). In $\mathrm{AC}$ welding system the supply is usually a single phase voltage at $50 \mathrm{~Hz}$. The supply circuit, shown in Fig. 1, is made by an AC supply system connected to an elevator current transformer through a couple of controlled diode. These diodes allow the regulation of the rms current value and so of the welding power through their duty cycle variation. Similarly in MFDC technique the primary current is regulated by a three phase inverter and the secondary current is rectified by a diode bridge (Fig. 2). The welding current versus time is reported in Fig. 3(a) for the AC and in Fig. 3(b) for the MFDC welding systems. From the environmental point of view, the comparison between the two technologies are very different. In fact while in the $\mathrm{AC}$ behavior the prevalent field component is at $50 \mathrm{~Hz}$, at
Table 1. Tissue properties used for Hugo model at $50 \mathrm{~Hz}$ (in $\mathrm{S} / \mathrm{m})$

\begin{tabular}{|c|c|c|}
\hline Tissue & $\sigma$ & $\omega \epsilon$ \\
\hline Bladder & 0.2054 & 0.0013 \\
\hline Blood & 0.7000 & 0.0000 \\
\hline Bones & 0.0201 & 0.0000 \\
\hline Cartilages & 0.1714 & 0.0046 \\
\hline Eye & 0.5027 & 0.0008 \\
\hline Fat Tissue & 0.0196 & 0.0041 \\
\hline Forecourt & 0.0827 & 0.0241 \\
\hline Gall Bladder & 0.9000 & 0.0000 \\
\hline Glands & 0.5214 & 0.0046 \\
\hline Gray Substance & 0.0753 & 0.0337 \\
\hline Intestine & 0.0545 & 0.0893 \\
\hline Intestine Contents & 0.5215 & 0.0057 \\
\hline Kidney & 0.0892 & 0.0281 \\
\hline Lens & 0.3214 & 0.0048 \\
\hline Liver & 0.0367 & 0.0051 \\
\hline Lung & 0.0684 & 0.0160 \\
\hline Marrow & 0.0016 & 0.0005 \\
\hline Mucous Membrane & 0.0004 & 0.0001 \\
\hline Nervus Opticus & 0.0274 & 0.0045 \\
\hline Neuronal Fabric & 0.0953 & 0.0337 \\
\hline Pancreas & 0.5214 & 0.0046 \\
\hline Skeleton Muscle & 0.2333 & 0.0493 \\
\hline Skin & 0.0002 & 0.0000 \\
\hline Spleen & 0.0857 & 0.0283 \\
\hline Stomach & 0.5214 & 0.0046 \\
\hline Ventricles & 0.0827 & 0.0241 \\
\hline White Substance & 0.0533 & 0.0147 \\
\hline
\end{tabular}


Table 2. Discretization data.

\begin{tabular}{ccccc}
\hline & \multicolumn{2}{c}{ Axisymmetric } & \multicolumn{2}{c}{ Hugo } \\
& tetrahedra & nodes & hexahedra & nodes \\
\hline mesh1 & 202674 & 37027 & 202627 & 226300 \\
mesh2 & 460006 & 82134 & 481244 & 523638 \\
mesh3 & 1426256 & 248280 & 1619305 & 1714837 \\
\hline
\end{tabular}

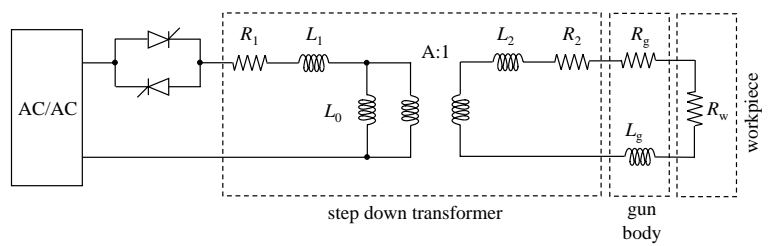

Fig. 1. Electrical equivalent circuit of an AC single phase resistance spot welding.

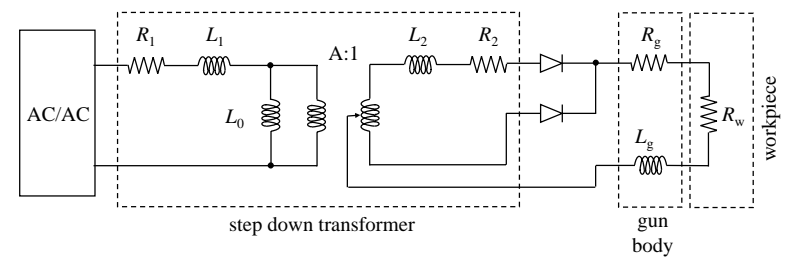

Fig. 2. Electrical equivalent circuit of an MFDC single phase resistance spot welding.

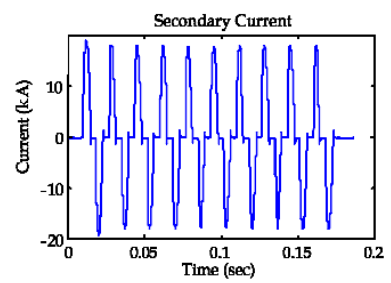

(a)

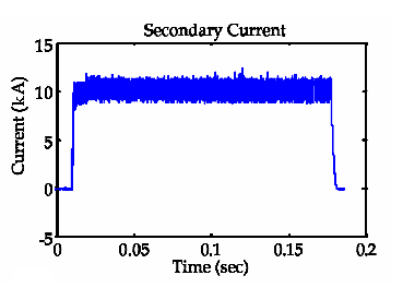

(b)
Fig. 3. Qualitative behavior of the secondary transformer current with (a) AC supply (b) MFDC supply.

MFDC the field is static. By considering the magnetic field limits at $50 \mathrm{~Hz}$ are strongly lower then those for the static case, the main attention to the worker exposure has to be paid for workers in proximity of AC welding systems.

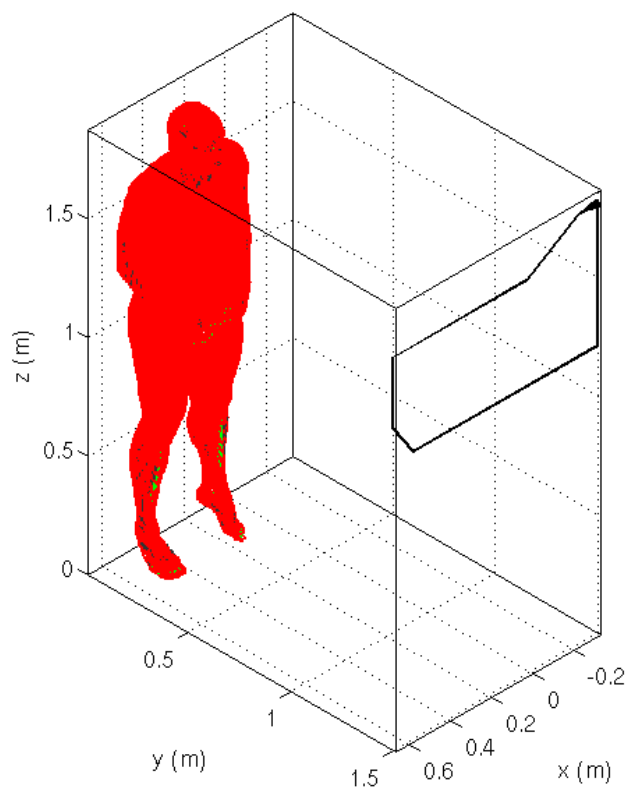

Fig. 4. Geometry under study with source position.

\section{Computational results}

The welding gun was placed at a distance of $1.5 \mathrm{~m}$ from the body as it can be seen in Fig. 4. Supply current value was set to $20 \mathrm{kA} \mathrm{rms}$ at the frequency of $50 \mathrm{~Hz}$. Firstly the value of magnetic flux density, created by the equipment at the body level, was computed and compared with the $a c$ tion values specified in the European Directive. The map of magnetic flux density absolute values inside the body is reported in Fig. 5 and, as it can be seen, these values reach are more than two times greater than the action values and an accurate evaluation of the maximum values of current density inside the body has been performed.

\subsection{Model comparison}

The first analysis on results has been devoted to the validation of the axisymmetric model. The geometrical resemblance of the axisymmetric model with the actual human 


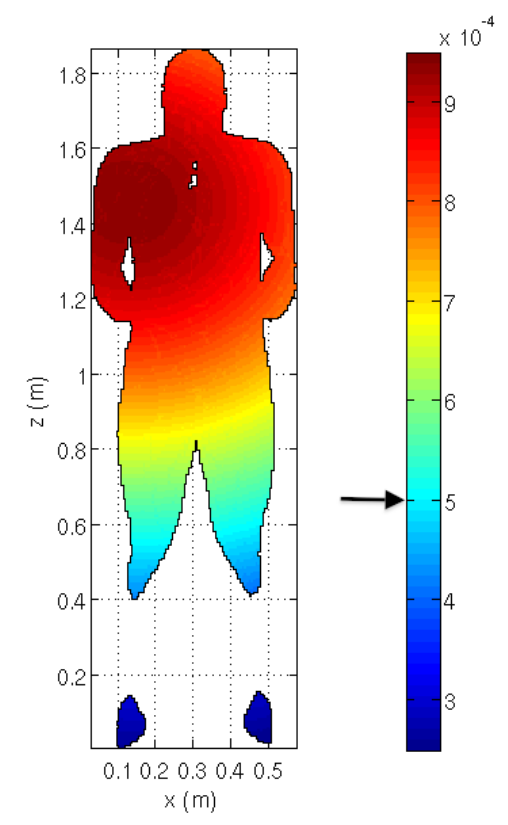

Fig. 5. Color map of magnetic flux density (in T). The value of $500 \mu \mathrm{T}$ is marked with an arrow.

body, allows to find correctly in which zones current densities are higher. In fact current density reaches its highest value at the neck level where conducting cross section is minimal, as it can be seen in Fig. 6. As far as dependence of results on discretization level is concerned, the maximum value of current density is weakly changing as function of the number of tetrahedra, as its maximum value varies from 9.01 to $10.1 \mathrm{~mA} / \mathrm{m}^{2}$ when mesh dimension changes from about 200 thousand to 1.5 million tetrahedra. This fact can be explained considering that, due to the material homogeneity of this model, also the coarsest mesh is able to solve the eddy current problem with adequate accuracy. The analysis of the problem with the Hugo model is by far more complex than the previous one. In fact in this case the realistic distribution of different tissues and organs inside the body can change abruptly its
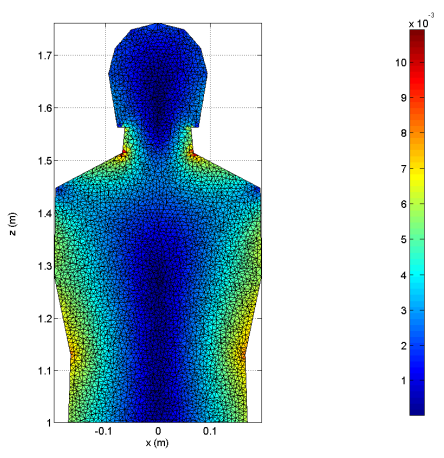

Fig. 6. Detail of induced eddy currents in axisymmetric model.

details passing from one discretization level to the other. For instance one tissue, which is present at the finest discretization level, can be absent in a coarser one. This is the case, for instance, of nervous tissues which is typically discontinuous in the $8 \mathrm{~mm}$ voxel resolution. This change in geometric distribution of tissues can justify why maximum value of current density greatly changes from one mesh to the other. Table 3 collects the maximum value of current densities in all tissues.

If geometric location of current density maximum value is considered, in all meshes it is located at the neck level, as it can be seen in Fig. 7. When evaluated with the Hugo model, the absolute value of current density is more than three times higher than that obtained by the axisymmetric model and also exceeding the exposure limit values indicated in the European Directive. It could be interesting to restrict the analysis to the nervous tissue and heart, since only short-term effects are under investigation. Table 4 summarizes these values. Nervous tissue includes white and grey substance, lens, nervus opticus and neuronal fabric. Heart includes forecourts, ventricles and the blood content. It is worth noting that the exposure limit 


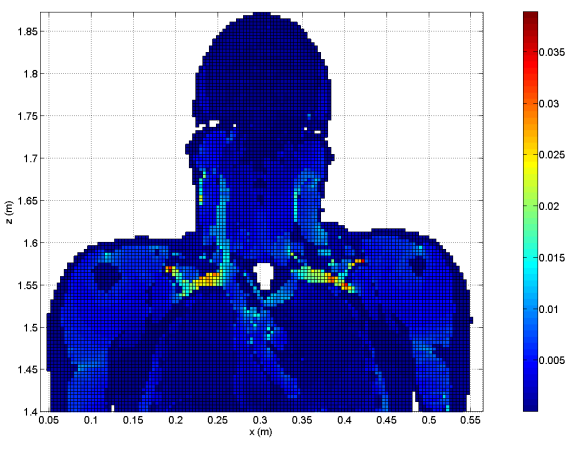

Fig. 7. Detail of induced eddy currents in Hugo model

of $10 \mathrm{~mA} / \mathrm{m}^{2}$ is not exceeded when limiting the study to the nervous tissue, which is taken as reference by ICNIRP for the definition of the safety limits for occupational exposure. The limit is violated in the heart, but the value of about $15 \mathrm{~mA} / \mathrm{m}^{2}$ is located in the blood content, thus it is difficult to define the real effect of this induced current.

\section{Conclusions}

In 2004 a European Directive has stated the reference levels for workers exposure to electromagnetic fields and, following studies performed by ICNIRP, has defined that at low frequency values, the induced eddy currents are the interaction mechanism between field and human body. Since this physical parameter is not directly measurable, other indicators are available to evaluate possible dangerous effects. Numerical solution of the eddy current problem induced inside the body is one of these.

The study carried out and described in this paper has highlighted that an accurate body model is needed to get a reliable estimate of the maximum value of induced eddy
Table 3. Maximum values of current densities for different discretizations in $\mathrm{mA} / \mathrm{m}^{2}$.

\begin{tabular}{|c|c|c|c|}
\hline & $4 \mathrm{~mm}$ & $6 \mathrm{~mm}$ & $8 \mathrm{~mm}$ \\
\hline Bladder & 6.0 & 5.5 & 5.2 \\
\hline Blood & 37.4 & 33.4 & 34.0 \\
\hline Bones & 5.3 & 4.8 & 4.2 \\
\hline Cartilages & 10.7 & 10.0 & 8.4 \\
\hline Eye & 2.4 & 3.2 & 2.7 \\
\hline Fat Tissue & 12.7 & 7.9 & 6.5 \\
\hline Forecourt & 2.9 & 2.2 & - \\
\hline Gall Bladder & 19.3 & 16.7 & 15.1 \\
\hline Glands & 17.0 & 16.9 & 17.0 \\
\hline Gray Substance & 2.5 & 2.1 & 1.7 \\
\hline Intestine & 6.9 & 4.4 & 3.7 \\
\hline Intestine Contents & 32.9 & 27.7 & 26.2 \\
\hline Kidney & 4.7 & 4.1 & 3.9 \\
\hline Lens & 1.2 & - & - \\
\hline Liver & 3.0 & 2.7 & 2.6 \\
\hline Lung & 5.6 & 5.0 & 5.2 \\
\hline Marrow & 0.5 & 0.3 & 0.3 \\
\hline Mucous Membrane & 0.03 & 0.02 & 0.02 \\
\hline Nervus Opticus & 1.6 & 1.5 & - \\
\hline Neuronal Fabric & 3.2 & 3.6 & 3.8 \\
\hline Pancreas & 10.7 & 8.5 & 7.7 \\
\hline Skeleton Muscle & 29.8 & 23.2 & 23.6 \\
\hline Skin & 0.2 & 0.1 & - \\
\hline Spleen & 4.7 & 3.7 & 3.8 \\
\hline Stomach & 12.8 & 10.8 & 10.6 \\
\hline Ventricles & 3.5 & 3.1 & 4.3 \\
\hline White Substance & 1.2 & 1.1 & 1.0 \\
\hline
\end{tabular}


Table 4. Maximum values of current densities for different discretizations in $\mathrm{mA} / \mathrm{m}^{2}$ : tissues of interest.

\begin{tabular}{lccc}
\hline & $4 \mathrm{~mm}$ & $6 \mathrm{~mm}$ & $8 \mathrm{~mm}$ \\
\hline Overall & 37.4 & 33.4 & 34.0 \\
Nervous Tissue & 3.2 & 3.6 & 3.8 \\
Heart & 15.5 & 16.7 & 4.3 \\
\hline
\end{tabular}

current. Simplified models, as the axisymmetric one used in this study, even if are able to get the most affected parts of the body, can give results which underestimate the values obtained with more accurate body models.

More accurate body models can give more reliable results but nevertheless can suffer from artificial geometrical discontinuities introduced by the discretization process. As for the Hugo model is concerned it arises, from the numerical experiments, that the $4 \mathrm{~mm}$ voxel discretization would be accurate enough to reasonably model all important tissues.

Work on this subject will be continued in order to find a reasonable trade off between computational cost of analyses and reliability of results.

\section{References}

1. D. of the European Parliament, of the Council of 29 April 2004, On the minimum health and safety requirements regarding the exposure of workers to the risks arising from physical agents (electromagnetic fields)

2. ICNIRP - International Commission on Non-Ionizing Radiation Protection, Health Physics 74, 494 (1998)

3. IEC Standard 62226-1:2004-11, Exposure to electric or magnetic fields in the low and intermediate frequency range
- methods for calculating the current density and internal electric field induced in the human body part 1: General (2004)

4. T. Dawson, K. Caputa, M. Suchly, Physics in Medicine and Biology 47, 2561 (2002)

5. E. Tonti, IEEE Transactions on Magnetics 38, 333 (2002)

6. F. Freschi, L. Giaccone, M. Repetto, COMPEL - The International Journal for Computation and Mathematics in Electrical and Electronic Engineering 27(6), 1343 (2008)

7. U.A.N. NATICK/TR-89/044, Anthropometric survey of us army personnel: methods and summary statistics (1988)

8. IEC Standard 62226-3-1:2007-05, Exposure to electric or magnetic fields in the low and intermediate frequency range - methods for calculating the current density and internal electric field induced in the human body. part 3-1: Exposure to electric fields - analytical and $2 d$ numerical models (2007)

9. F. Sachse, C. Werner, C. Waarden, O. Dossel, Computerized Medical Imaging and Graphics 24, 165 (2000)

10. K. Cole, R. Cole, Journal of Chemical Physics 1, 341 (1941)

11. S. Gabriel, R. Lau, C. Gabriel, Physics in Medicine and Biology 41, 2271 (1996) 